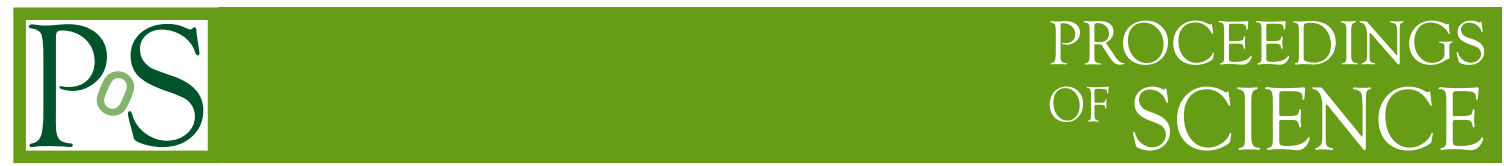

\title{
Virtual Photon Measurements with the HADES at GSI
}

\section{Jan-Hendrik Otto ${ }^{a, *}$ for the HADES collaboration}

a Justus-Liebig Universität Gießen,

Heinrich Buff Ring 16, 35392 Gießen, Germany

E-mail: jan.h.otto@physik.uni-giessen.de

In this work we present work in progress results on the dielectron analysis of the HADES Ag+Ag data at a centre-of-mass energy of $\sqrt{s_{N N}}=2.55 \mathrm{GeV}$. The high statistics data sample in combination with a strongly increased electron detection efficiency allows for a signal even in the $\Phi$-meson mass region. With the recent upgrade of the HADES RICH photodetector an unprecedented quality and signal-to-background ratio was achieved. The obtained dielectron signal spectrum is compared to the simulated hadronic cocktail spectrum at freeze out.

*** Particles and Nuclei International Conference - PANIC2021 ***

*** 5 - 10 September, $2021 * * *$

*** Online ***

${ }^{*}$ Speaker 


\section{Introduction}

The High Acceptance DiElectron Spectrometer (HADES) [1] is dedicated to the measurement of electromagnetic and rare hadronic [2] probes from elementary to heavy-ion collisions and to study the in-medium behaviour of dileptons, as their spectral distributions reveal the thermal properties of the medium.

In heavy-ion collisions at $\sqrt{s_{N N}}=2-3 \mathrm{GeV}$ as measured with HADES, matter is compressed to 2-3 times the groundstate density and heated up to temperatures of about $T=70 \mathrm{MeV}$ [3]. Their study allows to address the microscopic properties of the medium as present in e.g. neutron star mergers [4] located in the moderate temperature and high-density regime of the QCD phase diagram.

\section{The HADES experiment}

HADES is a fixed target experiment located at the SIS 18 accelerator at GSI, Germany. It is realized as a magnet spectrometer covering nearly the full forward hemisphere apart from small polar angles. Two layers of low mass Mini-Drift-Chambers (MDCs) located in front and two behind the field region provide tracking information. Time of flight measurement is performed by Resistive Plate Chambers (RPCs) and a ToF wall at polar angles of $18^{\circ}-44^{\circ}$ and $44^{\circ}-88^{\circ}$, respectively. A hadron blind Ring Imaging CHerenkov detector (RICH) enhances electron identification. The achieved particle identification for reconstructed tracks in Ag+Ag collisions at $\sqrt{s_{N N}}=2.55 \mathrm{GeV}$ is shown in fig. 1 , left.
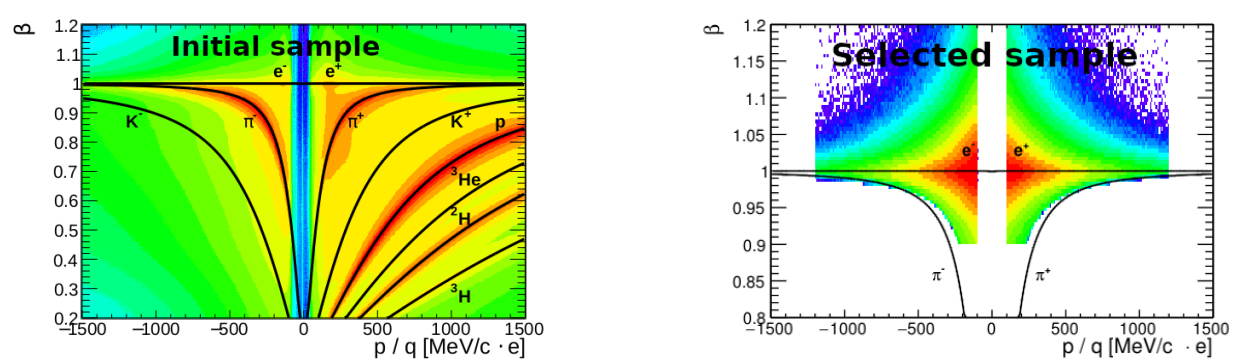

Figure 1: Left: Velocity vs. momentum distributions for reaction products in $\mathrm{Ag}+\mathrm{Ag}$ at $\sqrt{s_{N N}}=2.55 \mathrm{GeV}$ when combining tracking and time-of-flight information. The lines depict calculated values of $\beta$ vs. $p$ for particle species. Right: Track sample after applying electron selection criteria.

Recently, the RICH detector has been upgraded with a new photon detector consisting of 428 64-channel H12700 Multi-Anode PMTs (MAPMTs) with FPGA based readout [5]. Furthermore an electromagnetic calorimeter has been added covering only a fraction of the total HADES acceptance yet. In total, 4.5 billion $\mathrm{Ag}+\mathrm{Ag}$ collisions with a centrality selection of $0-40 \%$ are analyzed.

\section{Electron selection}

\subsection{Electron identification}

Electron identification is performed by matching reconstructed tracks with rings and time-offlight information. The velocity vs. momentum distribution of the resulting electron sample is 
shown in fig. 1, right. Pion misidentification arises from randomly matched pion tracks to RICH rings. The pion suppression factor (fig. 2, left) is defined as the ratio of initially produced pions to pions that are present in the track sample after applying electron selection criteria to a simulated sample. It is in the order of $\pi_{\text {sup }} \sim 10^{5}$ and even higher for $p<400 \mathrm{MeV} / \mathrm{c}$.
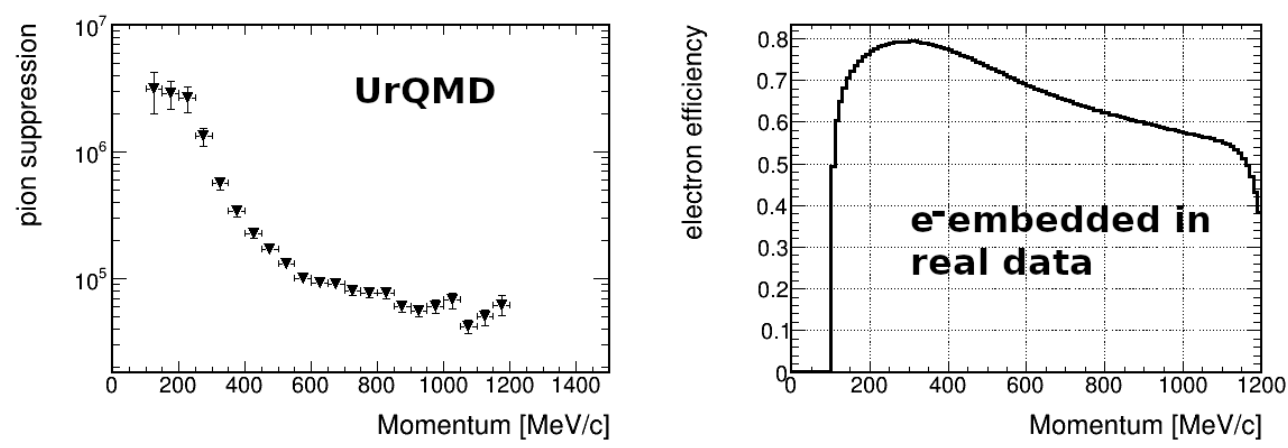

Figure 2: Left: Momentum dependent pion suppression factor derived from simulations using UrQMD as event generator. The errors shown are of statistical nature only. Right: Momentum dependent electron detection efficiency extracted from electrons embedded into real data.

The electron detection efficiency (within the acceptance) is derived by embedding simulated electrons with momenta up to $p_{e}=1.5 \mathrm{GeV} / \mathrm{c}$ into real data. It exceeds $\epsilon=50 \%$ over the whole momentum range and reaches up to $\epsilon \sim 80 \%$ at small momenta (fig. 2, right). An electron purity estimation in simulations based on the event generator UrQMD [6] and an experimental RICHrotation technique yield $P>99 \%$ at low and $P \approx 90 \%$ at high momentum which will be further improved with a fully equipped and properly calibrated electromagnetic calorimeter in future.

\subsection{Conversion suppression}

The dominant background source in a high purity dielectron sample arises from partially reconstructed conversion pairs. Due to the high level performance of the RICH detector these can be efficiently rejected using ring information only. Ring finding based on Hough-Transformation [7] can separate rings with opening angles starting at around $\alpha \sim 2^{\circ}$ (fig. 3, middle) exceeding the double-track separation of the MDCs. Double rings with even smaller opening angle can still be identified by counting the amount of Cherenkov photons in the vicinity of a fitted ring. The double ring in fig. 3, right, e.g. consists of $N=26$ detector hits and has to be compared to the average amount of $N=16$ for single rings (see fig. 3, left). Applying a cut on the amount of Cherenkov photons, the conversion contamination in the electron sample can be significantly reduced.

\section{Electron Pair Formation}

In the pairing of electrons and positrons, combinatorial background arises which can be described by the geometrical mean of same-event $e^{+} e^{+}$and $e^{-} e^{-}$pairs $\left(\left\langle F G_{++}\right\rangle,\left\langle F G_{--}\right\rangle\right)$weighted with the so called $k$-factor. This accounts for acceptance differences in the pairings and is derived using the event-mixing technique, see formula 1 [8]. In event-mixing electrons and positrons reconstructed in different events are paired $\left(\left\langle f g_{+-}\right\rangle,\left\langle f g_{++}\right\rangle,\left\langle f g_{--}\right\rangle\right)$. This allows for almost unlimited statistics. 

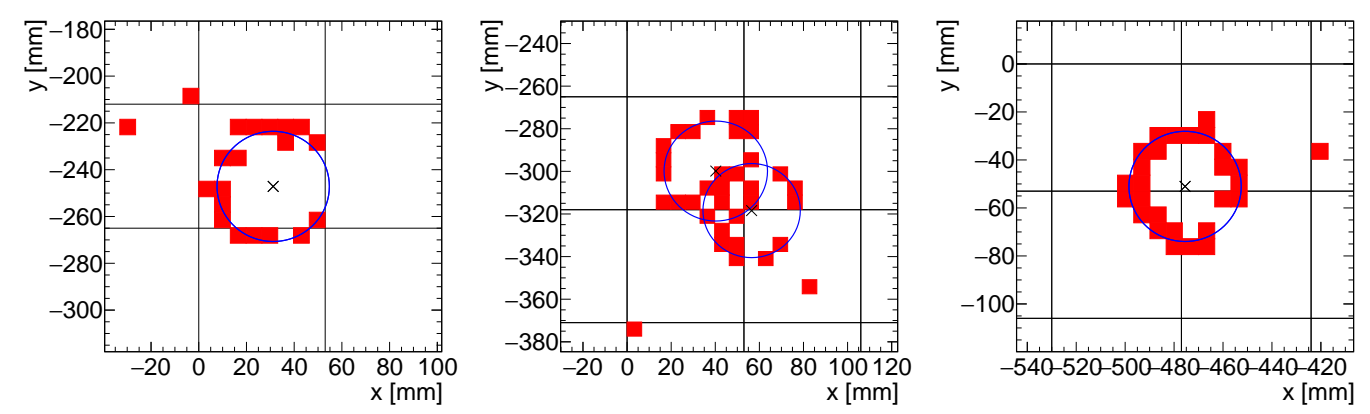

Figure 3: Zoom-in views of the RICH MAPMT plane. Red squares correspond to detected Cherenkov photons that are fitted to rings (blue circles). Black lines indicate MAPMT boarders. Left: A single ring. Centre: Two reconstructed rings with an opening angle of about $\alpha \sim 2^{\circ}$. Right: Cherenkov photon distributions of a close conversion track pair leading to overlapping rings.

$$
\left\langle B G_{+-}\right\rangle=k \cdot 2 \sqrt{\left\langle F G_{++}\right\rangle\left\langle F G_{--}\right\rangle} \quad \text { with } \quad k=\frac{\left\langle f g_{+-}\right\rangle}{2 \sqrt{\left\langle f g_{++}\right\rangle\left\langle f g_{--}\right\rangle}}
$$
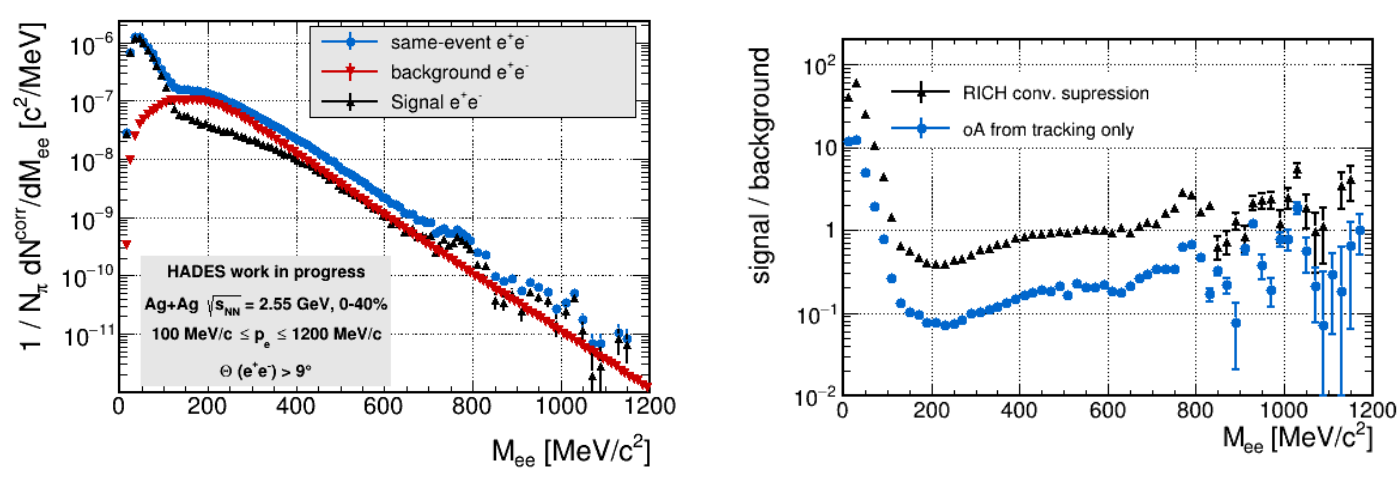

Figure 4: Left: Efficiency corrected invariant mass spectrum of dielectrons in the $0-40 \%$ most central $\mathrm{Ag}+\mathrm{Ag}$ collisions at $\sqrt{s_{N N}}=2.55 \mathrm{GeV}$ inside the HADES acceptance. Right: S/B ratio using the RICH conversion suppression (black) and using close pair rejection from tracking only (blue).

This same-event like-sign combinatorial background evaluation is used for low invariant masses $M_{e e}<300 \mathrm{MeV} / \mathrm{c}^{2}$. For large masses beyond $M_{e e}>300 \mathrm{MeV} / \mathrm{c}^{2}$ the background is obtained from mixed event only in order to reduce statistical errors. Normalization to the same event background is obtained in the region $300 \mathrm{MeV} / \mathrm{c}^{2}<M_{e e} \leq 700 \mathrm{MeV} / \mathrm{c}^{2}$. The resulting efficiency corrected invariant mass distribution is shown in fig. 4, left. It is restricted to single lepton momenta of $100 \mathrm{MeV} / \mathrm{c}<p_{e}<1200 \mathrm{MeV} / \mathrm{c}$ and pair opening angles $\alpha>9^{\circ}$. At the $\rho, \omega$-mesons pole mass a clear enhancement above the exponential slope is visible and pairs up to the $\phi$-meson pole mass are reconstructed. The high statistics measurement allows for a differential analysis in e.g. centrality and pair momentum.

The extracted signal-to-background ratio is shown in the right panel of fig. 4. It surpasses $S / B=1$ for $M_{e e}>400 \mathrm{MeV} / \mathrm{c}^{2}$ and reaches up to $S / B \sim 3$ at around the $\omega$-meson pole mass. The $S / B$ values not using the purely RICH based double ring recognition are a factor of $\sim 5$ lower. 


\section{Hadronic decomposition of the dielectron spectrum}

To reveal eventual fireball medium contributions to the reconstructed spectrum of dielectrons, simulations of the relevant hadronic sources are performed utilizing the software package Pluto [9] (see fig. 5). A clear excess at invariant masses beyond the $\pi^{0}$ Dalitz contribution is observed. The expected $\pi^{0}$ and $\eta$ multiplicities are extrapolated from previous TAPS measurements [10]. From high momentum data an extrapolation to the $\omega$ and $\phi$ multiplicities is performed. Still missing in the cocktail presented in fig. 5 are contributions like bremsstrahlung originating from direct pp and pn interactions. The analysis of $\pi^{0}$ and $\eta$ production in the present $\mathrm{Ag}+\mathrm{Ag}$ data sample is in progress and will soon deliver measured values without the need for extrapolations thus allowing for the extraction of the excess yield.

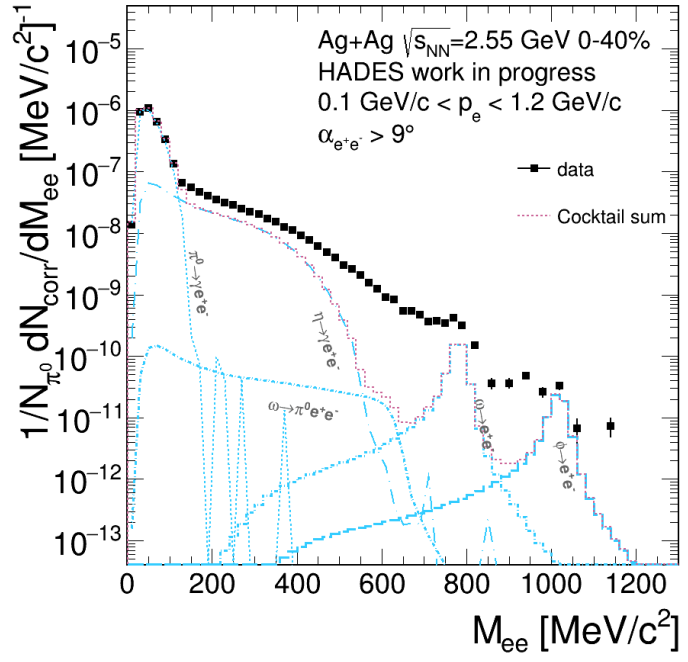

Figure 5: Composition of the dielectron signal into its hadronic cocktail contributions. For details see text.

\section{Summary}

We have shown work in progress results on the dielectron analysis in $\mathrm{Ag}+\mathrm{Ag}$ collisions at $\sqrt{s_{N N}}=2.55 \mathrm{GeV}$ measured with HADES. The upgraded detector allows for high efficient electron identification combined with high pion suppression and conversion recognition. This results in dielectron spectra of unprecedented quality in terms of statistics and S/B ratio. A differential analysis e.g. in centrality and also pair momentum is possible and currently in progress.

\section{References}

[1] G. Agakishiev et. al. (HADES), Eur. Phys. J. A41 (2009), pp. 243-277, http://dx. doi . org/10.1140/epja/i2009-10807-5, http://arxiv.org/abs/2004.10268

[2] Adamczewski-Musch, J. and others, Phys. Lett. B 778 (2018), pp. 403-407, https: //doi . org/10.1016/j.physletb.2018.01.048, https://arxiv.org/abs/1703.08418

[3] The HADES Collaboration, Nat. Phys. 15, 1040-1045 (2019), https : //doi . org/10 . 1038/ s41567-019-0583-8

[4] Matthias Hanauske et. al. J. Phys.: Conf. Ser. 878012031 (2017), https://doi.org/10. 1088/1742-6596/878/1/012031

[5] C. Pauly et. al. The 9th international workshop on Ring Imaging Cherenkov Detectors (RICH2016), https://doi .org/10.1016/j .nima.2017.02.067,

[6] Bass, S. A. and others, Prog. Part. Nucl. Phys. 41 (1998), pp. 255-369 https: //doi .org/ 10.1016/S0146-6410(98)00058-1, https://arxiv.org/abs/nucl-th/9803035

[7] S. Lebedev and G.Ososkov, Phys. Part.Nucl. Lett.6(2009), pp.161-176, https: //doi .org/ $10.1134 /$ S1547477109020095 
[8] A. Adare et. al. (PHENIX), Phys. Rev. C81, 034911 (2010), https://doi .org/10 . 1103/ PhysRevC.81.034911, https://arxiv.org/abs/0912.0244

[9] Fröhlich, I. and others, PoS ACAT 076 (2007), https: //doi .org/10.22323/1.050.0076, https://arxiv.org/abs/0708.2382v2

[10] R. Holzmann et. al. (TAPS), Acta Phys. Polon. B29 (1998), pp. 3051-3054 